\title{
Lessons Learned From Field Deployment of the Hemaapp: A Non-Invasive Hemoglobin Measurement tool
}

\author{
Christopher M Westgard ${ }^{1 *}$ and Edward J Wang ${ }^{2}$ \\ ${ }^{1}$ Department of Research and Evaluation, Asociacion Red Innova, USA \\ ${ }^{2}$ Department of Electrical Engineering, University of Washington, USA
}

Received: November 30, 2017; Published: December 06, 2017

*Corresponding author: Christopher M Westgard, Department of Research and Evaluation, Asociacion Red Innova, Lima, Peru, Elementos, Perú, USA; Email: cmwestgard@gmail.com

\section{Introduction}

The Hemaapp [1] is a smart phone application that measures the hemoglobin level in your finger by using the phone's built-in camera and flash. The hemoglobin measurement is done noninvasively, by placing the finger of the patient on the camera of the phone. The light from the flash allows the camera to record the absorption of light by the blood, while the Hemaapp application analyzes the images to determine the level of hemoglobin. The application was developed at the University of Washington's Ubiquitous Computing Laboratory. The University of Washington team worked with the NGO, Asociacion Red Innova, to test the innovative technology in the field. The team conducted a field deployment in a variety of diverse settings in Peru, including two communities in the Amazon region, an urban-slum community in Lima, and three clinics in Lima. The following report was drafted to reflect the lessons learned in the field.

Participants of the study received an examination with the Hemaapp and then an examination with an invasive method to measure hemoglobin, such as the HemoCue or Complete Blood Count. The results of the two tests were compared to improve the precision of the algorithm of the Hemaapp. 100\% of participants indicated that they prefer the analysis by the Hemaapp to an analysis by the HemoCue or CBC. In additional to a satisfaction questionnaire, the research team identified a number of opportunities to improve the diagnostic tool and methods for campaigns that utilize the Hema App.

Some of the findings included:

a) A cloth strip can be installed on the phone case to help hold the finger securely over the camera. This will help the participant from moving excessively during the analysis and ensure that the technician properly placed the subject's finger over the camera. The cloth strip also serves to block out the ambient light to ensure the light is stable and consistent in all environments. b) It can be difficult to conduct the Hemaapp measurement on very young children. Some of children in the field study were afraid or uncomfortable about placing their finger on the camera for 15 seconds. As Hemaapp relies on optical measurements through the finger's skin, if the subject does not hold their finger still over the camera, the excessive motion will render the measurement unusable. Thus, Hemaapp is not suitable for crying children who are resisting. For this reason, we found that it is important to take a moment to reassure the child and gain their confidence. This can be accomplished more easily by showing a video with a cell phone. A future edition of the HemaApp may include a child-friendly video that convinces the child to put their finger on the camera and then continues for 15 seconds to ensure the child does not move during the analysis.

c) A phone case that is appealing to children may also be beneficial for calming the child and encouraging them to participate. Some of the children seemed particularly frightened by the black phone case, but became noticeably less agitated when we showed them the screen display of the phone.

d) We found that children are calmer when sitting in a chair independently rather than on an adult's lap during the measurement. The independence may reduce the amount of anxiety caused by a lack of control.

e) It is often not feasible to examine children under the age of 10 months with the Hemaapp as their fingers are too small to cover the camera and the flash. An attachment may be constructed in the future to utilize with babies less than 1 year of age.

f) The flash of the camera can get hot. It is best to wait until the finger is placed on the camera, secured with a cloth strip, and then placed securely on a table before turning on the flash, thus minimizing the amount of time the flash is illuminated. 
When the device is used repeatedly and rapidly, cleaning the flash and camera with alcohol can help cool the light.

g) Participants in the clinics were less willing to participate in the study with the innovative tool than participants in the community. Those in the clinic were more cautious of participating in the study. Those in the communities were excited at the prospect of trying an innovative tool and receiving the exam with the cell phone.

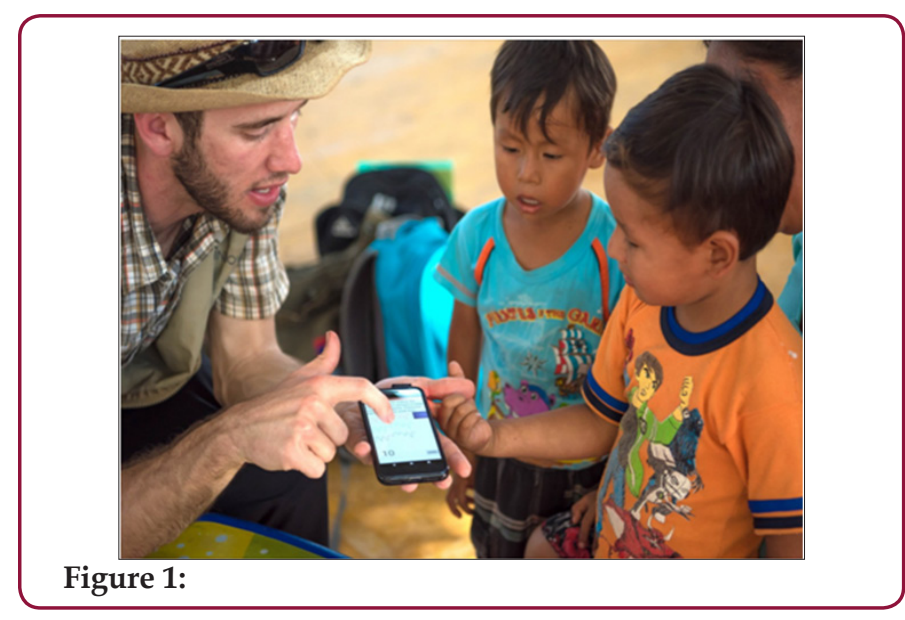

Through the field deployment in Peru we learned several lessons for how to improve the overall effectiveness of the diagnostic tool. Simple modifications of a phone case and steps taken between exams can increase the appeal and effectiveness of the tool, especially when dealing with children. The team at the University of Washington is continuously making improvements to the application and the device to make it more precise and userfriendly. We are continuing to address the challenges identified in the Peru trials to continuously improve the prototype and learn from the end-user. The team at Asociacion Red Innova is planning the next field validation campaign for the Hemaapp with great consideration to the lessons learned from the last deployment. We hope to make the evaluation more appealing to children and streamlining the procedure to improve the efficiency of the measurement. Based on the satisfaction we observed from the recipients of the Hemaapp we know that the tool is highly desired and feasible to execute in large scale anemia screening campaigns in diverse settings. Most of the children and their parents in the Amazonian and urban communities were excited to receive the evaluation with the cell phone. We believe the appeal of the technology represents a great opportunity to make more tools like the Hemaapp for diagnosis, surveillance, and education to improve public health in the future.

\section{References}

1. EJ Wang, W Li, J Zhu R Rana, SN Patel (2017) Noninvasive hemoglobin measurement using unmodified smartphone camera and white flash. Seogwipo pp. 2333-2336.

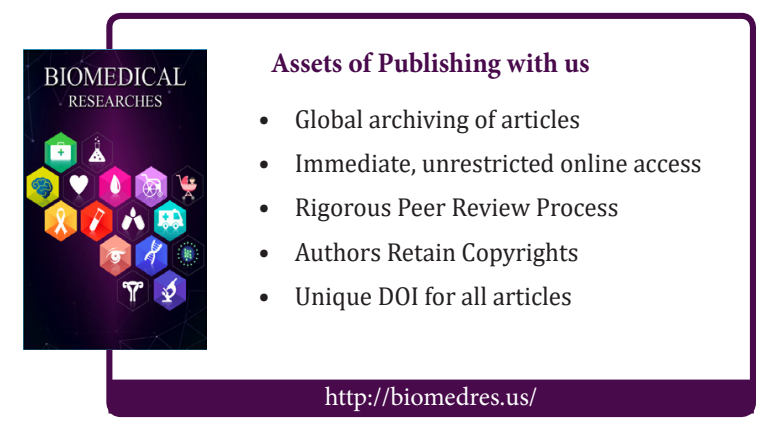

